\title{
Multipole-based distance-dependent screening of Coulomb integrals
}

\author{
Cite as: J. Chem. Phys. 151, 084111 (2019); doi: 10.1063/1.5111054 \\ Submitted: 22 May 2019 - Accepted: 7 August 2019 • Published Online: 29 August 2019
}

Andreas Irmler ${ }^{1, a)}$ (D) and Fabian Pauly ${ }^{2,3}$ (D)

\begin{abstract}
AFFILIATIONS
${ }^{1}$ Institute for Theoretical Physics, TU Wien, Wiedner Hauptstraße 8-10/136, 1040 Vienna, Austria

${ }^{2}$ Okinawa Institute of Science and Technology Graduate University, Onna-son, Okinawa 904-0495, Japan

${ }^{3}$ Department of Physics, University of Konstanz, 78457 Konstanz, Germany
\end{abstract}

a) Electronic mail: andreas.irmler@tuwien.ac.at

\begin{abstract}
We derive a new estimate for two-electron repulsion integrals (ERIs), when evaluated within a local atomic basis set. It is based on the multipole expansion and provides a rigorous upper bound of an ERI for well-separated charge distributions. The scheme is generally applicable in any formalism that uses ERIs. We employ it here to screen for potentially negligible contributions in the calculation of the Fock exchange matrix. Using Gaussian basis functions, we show that the estimate allows us to accelerate the construction of the exchange matrix by up to a factor of two without introducing further approximations.
\end{abstract}

Published under license by AIP Publishing. https://doi.org/10.1063/1.5111054

\section{INTRODUCTION}

In Hartree-Fock calculations with local basis functions, it is crucial to screen for negligible electron repulsion integral (ERI) contributions in the construction of the Fock exchange matrix, since the calculation of two-electron integrals is usually the time-determining step. Taking into account the locality of the basis set, the number of significant Fock exchange matrix contributions increases as $\mathcal{O}\left(N^{2}\right)$, with $N$ being the number of basis functions that are used to describe the system. This is evident from the expression for the exchange matrix

$$
K_{\mu \lambda}=\sum_{v \kappa} D_{v \kappa}(\mu v \mid \kappa \lambda),
$$

the definition of the ERIs

$$
(\mu \nu \mid \kappa \lambda)=\iint d^{3} r_{1} d^{3} r_{2} \frac{\phi_{\mu}^{*}\left(\mathbf{r}_{1}\right) \phi_{v}\left(\mathbf{r}_{1}\right) \phi_{\kappa}^{*}\left(\mathbf{r}_{2}\right) \phi_{\lambda}\left(\mathbf{r}_{2}\right)}{\left|\mathbf{r}_{1}-\mathbf{r}_{2}\right|},
$$

and the fact that the local basis functions $\phi_{\mu}(\mathbf{r})$ and $\phi_{\nu}(\mathbf{r})$ show negligible overlap, if they are spatially separated.

A well-established screening of ERIs uses the Schwarz inequality

$$
(\mu \nu \mid \kappa \lambda) \leq(\mu \nu \mid \mu \nu)^{1 / 2}(\kappa \lambda \mid \kappa \lambda)^{1 / 2},
$$

proposed by Häser and Ahlrichs, ${ }^{2}$ which represents a rigorous upper bound and is in addition very convenient to calculate. A drawback of the Schwarz estimate is, however, that it does not account for the separation between left $(\mid \mu v))$ and right $(\mid \kappa \lambda))$ charge distributions, as it is apparent from the factorization in Eq. (3). Consequently, the Schwarz screening overestimates ERIs, in which left and right charge distributions are well-separated (WS).

The fast multipole method attracted much attention in the field of electronic structure theory, when formulated with local basis functions. The seminal work of White et al. ${ }^{3,4}$ adapted the fast multipole method, originally developed for treating point charges, ${ }^{5}$ to continuous local charge distributions. The central idea is that the Coulomb interaction I of two normalized s-type Gaussian functions, taken to be the left and right charge distributions of an ERI, reads

$$
I=\frac{1}{R} \operatorname{erf}\left(\sqrt{\frac{\alpha_{p} \alpha_{q}}{\alpha_{p}+\alpha_{q}}} R\right),
$$

with the Gaussian exponents $\alpha_{p}$ and $\alpha_{q}$ and the distance $R$ between the distributions. ${ }^{4}$ Since the error function rapidly approaches 1 with increasing argument, the interaction of two WS Gaussian functions can be accurately described by the multipole approximation. Equation (4) allows us to introduce an extent of the Gaussian distribution as

$$
r_{\mathrm{ext}, p}=\alpha_{p}^{-1 / 2} \operatorname{erfc}^{-1}\left(\epsilon_{\mathrm{o}}\right)
$$


where $\epsilon_{\mathrm{o}}$ represents the overlap threshold that controls the desired accuracy. Gaussian distributions with a center-to-center distance

$$
R>r_{\mathrm{ext}, p}+r_{\mathrm{ext}, q}
$$

are classified as WS. A detailed derivation and discussion of relations (4) - (6) can be found in the book of Helgaker.

Unfortunately, a fast multipole scheme cannot be formulated for the exchange part, as already pointed out by Burant et al. ${ }^{7}$ Instead the multipole approximation can be utilized to compute individual ERIs efficiently, as already reported. ${ }^{8-10}$ Furthermore, the multipole approximation was used for the derivation of an integral screening scheme by Lambrecht and Ochsenfeld. ${ }^{11,12}$ Although yielding a rigorous upper bound estimate, Ochsenfeld et al. recommended a subsequently developed screening scheme. ${ }^{13}$ The latter appears as much tighter bound but loses the rigorous upper bound quality. Along the same lines, Hollman et al. ${ }^{14}$ presented a distance-dependent screening for three-center Coulomb integrals occurring in density fitting approximations. Most recently, further integral estimates were presented by Thompson and Ochsenfeld ${ }^{15}$ with the added value of being applicable to general two-electron operators such as those found in explicitly correlated theories and in short-range hybrid density functionals.

Finally, there are completely different approaches to evaluate the exchange matrix more efficiently. They comprise the resolution of the identity method applied to the exchange, ${ }^{16}$ also known as RI-K, or the "chain of sphere" exchange algorithm (COSX). ${ }^{17} \mathrm{~A}$ comparison of RI-K and COSX can be found in Ref. 18 .

Our work is structured as follows: First, we derive a general ERI estimate based on the multipole approximation in Sec. II, before we analyze the developed screening scheme numerically in Sec. III. In Sec. IV, we apply it to specific systems and study the performance. Finally, we conclude in Sec. V.

\section{METHODOLOGY}

\section{A. Multipole-based estimate for ERIs}

For the present formulation, we use the scaled regular and irregular solid harmonics, ${ }^{6}$ which are defined by

$$
\begin{aligned}
O_{l m}(\mathbf{r}) & =\frac{|\mathbf{r}|^{l}}{\sqrt{(l+m) !(l-m) !}} C_{l m}(\theta, \phi), \\
M_{l m}(\mathbf{r}) & =\frac{\sqrt{(l+m) !(l-m) !}}{|\mathbf{r}|^{l+1}} C_{l m}(\theta, \phi) .
\end{aligned}
$$

The functions

$$
C_{l m}(\theta, \phi)=\sqrt{\frac{(l-m) !}{(l+m) !}} P_{l m}(\cos \theta) e^{i m \phi}
$$

are the spherical harmonics with Racah's normalization. We follow the phase convention of Condon and Shortley, ${ }^{19}$ and the Legendre polynomials are obtained from Rodrigues' formula

$$
P_{l m}(x)=(-1)^{m} \frac{\left(1-x^{2}\right)^{m / 2}}{2^{l} l !} \frac{d^{l+m}}{d x^{l+m}}\left(x^{2}-1\right)^{l}
$$

which holds for $0 \leq m \leq l$. Polynomials with negative sign are defined via

$$
P_{l-m}(x)=(-1)^{m} \frac{(l-m) !}{(l+m) !} P_{l m}(x) .
$$

Exploiting the relations introduced, the inverse distance can be written as

$$
\frac{1}{|\mathbf{R}-(\mathbf{a}+\mathbf{b})|}=\sum_{l=0}^{\infty} \sum_{m=-l}^{l} \sum_{j=0}^{\infty} \sum_{k=-j}^{j} O_{l m}(\mathbf{a}) M_{j+l, m+k}^{*}(\mathbf{R}) O_{j k}(\mathbf{b}) .
$$

The rotation of spherical harmonics can be expressed by the Wigner $D$-matrix ${ }^{20}$

$$
C_{l m}\left(\theta^{\prime}, \phi^{\prime}\right)=\sum_{k=-l}^{l} D_{k m}^{l}(\alpha, \beta, \gamma) C_{l k}(\theta, \phi)
$$

The functional arguments of the elements of the Wigner $D$-matrix are the three Euler angles $\alpha, \beta$, and $\gamma$, and $\theta^{\prime}$ and $\phi^{\prime}$ are the polar and azimuthal angles in the rotated coordinate system. Note that a rotation does not mix elements with different $l$.

Independent of the rotation of the coordinate system, the following invariant is found:

$$
\begin{aligned}
\sum_{m=-l}^{l}\left|C_{l m}\left(\theta^{\prime}, \phi^{\prime}\right)\right|^{2}= & \sum_{m=-l}^{l} \sum_{k=-l}^{l} \sum_{k^{\prime}=-l}^{l} D_{k m}^{l *}(\alpha, \beta, \gamma) \\
& \times C_{l k}^{*}(\theta, \phi) D_{k^{\prime} m}^{l}(\alpha, \beta, \gamma) C_{l k^{\prime}}(\theta, \phi) \\
= & \sum_{k=-l}^{l}\left|C_{l k}(\theta, \phi)\right|^{2},
\end{aligned}
$$

which holds due to the property of the Wigner $D$-matrices ${ }^{20}$

$$
\sum_{n=-l}^{l} D_{m n}^{l *}(\alpha, \beta, \gamma) D_{m^{\prime} n}^{l}(\alpha, \beta, \gamma)=\delta_{m m^{\prime}} .
$$

The scaled regular solid harmonics of Eq. (7) transform under rotation as

$$
O_{l m}\left(\mathbf{r}^{\prime}\right)=\sum_{k=-l}^{l} \frac{\sqrt{(l-k) !(l+k) !}}{\sqrt{(l-m) !(l+m) !}} D_{k m}^{l}(\alpha, \beta, \gamma) O_{l k}(\mathbf{r}) .
$$

In analogy to Eq. (14), we introduce the following rotationally invariant functions for the scaled regular solid harmonics:

$$
\bar{O}_{l m}(r)=\delta_{0 m} \frac{1}{l !} \sqrt{\sum_{m=-l}^{l}(l+m) !(l-m) !\left|O_{l m}(\mathbf{r})\right|^{2}},
$$

which are independent of the orientation of the coordinate system, i.e., they are functions of $r=|\mathbf{r}|$. Since $\bar{O}_{l m}(r)$ vanishes by definition for all $m \neq 0$, we suppress this index and write $\bar{O}_{l}(r)=\bar{O}_{l 0}(r)$ in the following.

Exploiting Eq. (17), an upper bound of Eq. (12) can be found as

$$
\frac{1}{|\mathbf{R}-(\mathbf{a}+\mathbf{b})|} \leq \sum_{l=0}^{\infty} \bar{O}_{l}(a) \sum_{j=0}^{\infty} \frac{(l+j) !}{R^{l+j+1}} \bar{O}_{j}(b)
$$

by using the maximum possible value of $M_{l 0}(\mathbf{R})$ at $\theta=0$ and $\phi=0$. The equality holds, if $\mathbf{R}, \mathbf{a}$, and $\mathbf{b}$ are all aligned in parallel and if the vectors $\mathbf{a}$ and $\mathbf{b}$ both point in the opposite direction of $\mathbf{R}$. 
For all other alignments of the three vectors, the right-hand side is an upper bound of the left-hand side of the equation.

The ERI can be expressed as

$$
(\mu \nu \mid \kappa \lambda)=\sum_{l=0}^{\infty} \sum_{m=-l}^{l} \Omega_{l m}^{\mathbf{a}} \sum_{j=0}^{\infty} \sum_{k=-j}^{j} M_{l+j, m+k}(\mathbf{R}) \Omega_{j k}^{\mathbf{b}}+\epsilon,
$$

with the multipole moment of the left charge distribution

$$
\Omega_{l m}^{\mathrm{a}}=\int d^{3} r \phi_{\mu}^{*}(\mathbf{r}) \phi_{v}(\mathbf{r}) O_{l m}(\mathbf{r})
$$

and an analogous expression for the moment of the right charge distribution $\Omega_{j k}^{\mathbf{b}}$. Here, $\mathbf{a}$ and $\mathbf{b}$ are the centers of multipole expansion of the left and right charge distributions, respectively, and the vector $\mathbf{R}$ is the connection vector between these two expansion centers. As mentioned before, the multipole approximation holds only for nonoverlapping distributions. Equation (19) defines the overlap error $\epsilon$ as the deviation of the exact value of the ERI from the result, obtained with the multipole approximation summed up to infinite order of angular momentum quantum number $l$.

Along the lines leading to $\bar{O}_{l}(r)$, we define the $m$-independent multipole moment or "multipole norm"

$$
\bar{\Omega}_{l}^{\mathbf{a}}=\frac{1}{l !} \sqrt{\sum_{m=-l}^{l}(l+m) !(l-m) !\left|\Omega_{l m}^{\mathbf{a}}\right|^{2}}
$$

and thereby obtain the following inequality for the ERI:

$$
|(\mu \nu \mid \kappa \lambda)| \leq \sum_{l=0}^{\infty} \sum_{j=0}^{\infty} \bar{\Omega}_{l}^{\mathbf{a}} \frac{(l+j) !}{R^{l+j+1}} \bar{\Omega}_{j}^{\mathbf{b}}+\epsilon .
$$

No manageable expression for estimating the size of the ERI has been obtained yet due to the infinite sums over angular momentum quantum numbers $l$ and $j$ in Eq. (22) and the a priori unknown form of the expressions $\bar{\Omega}_{l}^{\mathrm{a}}$. We seek to fulfill an inequality of the form

$$
\bar{\Omega}_{l}^{\mathbf{a}} \leq \bar{\Omega} \frac{\mathbf{a}}{l !} \frac{r_{\mathrm{e}, \mathbf{a}}^{l}}{l !}
$$

If the parameters $\bar{\Omega}^{\mathbf{a}}$ and $r_{\mathrm{e}, \mathbf{a}}$ are chosen such that the inequality is fulfilled for every order $l=0, \ldots, \infty$, Eq. (22) simplifies to

$$
\begin{aligned}
|(\mu \nu \mid \kappa \lambda)| & \leq \bar{\Omega}^{\mathbf{a}} \bar{\Omega}^{\mathbf{b}} \sum_{l=0}^{\infty} \sum_{j=0}^{\infty} \frac{r_{\mathrm{e}, \mathbf{a}}^{l}}{l !} \frac{(l+j) !}{R^{l+j+1}} \frac{r_{\mathrm{e}, \mathbf{b}}^{j}}{j !}+\epsilon \\
& =\bar{\Omega}^{\mathbf{a}} \bar{\Omega}^{\mathbf{b}} \frac{1}{R-r_{\mathrm{e}, \mathbf{a}}-r_{\mathrm{e}, \mathbf{b}}}+\epsilon .
\end{aligned}
$$

A tighter bound may be obtained by writing the first terms of Eq. (24) explicitly as

$$
\begin{aligned}
|(\mu \nu \mid \kappa \lambda)| \leq & \bar{\Omega}_{0}^{\mathbf{a}} \frac{1}{R} \bar{\Omega}_{0}^{\mathbf{b}}+\bar{\Omega}_{1}^{\mathbf{a}} \frac{1}{R^{2}} \bar{\Omega}_{0}^{\mathbf{b}}+\bar{\Omega}_{0}^{\mathbf{a}} \frac{1}{R^{2}} \bar{\Omega}_{1}^{\mathbf{b}}+\bar{\Omega}^{\mathbf{a}} \bar{\Omega}^{\mathbf{b}} \\
& \times\left(\sum_{l=2}^{\infty} \frac{r_{\mathrm{e}, \mathbf{a}}^{l}}{R^{l+1}}+\sum_{j=2}^{\infty} \frac{r_{\mathrm{e}, \mathbf{b}}^{j}}{R^{j+1}}+\sum_{l=1}^{\infty} \sum_{j=1}^{\infty} \frac{r_{\mathrm{e}, \mathbf{a}}^{l}}{l !} \frac{(l+j) !}{R^{l+j+1}} \frac{r_{\mathrm{e}, \mathbf{b}}^{j}}{j !}\right)+\epsilon \\
= & \bar{\Omega}_{0}^{\mathbf{a}} \frac{1}{R} \bar{\Omega}_{0}^{\mathbf{b}}+\bar{\Omega}_{1}^{\mathbf{a}} \frac{1}{R^{2}} \bar{\Omega}_{0}^{\mathbf{b}}+\bar{\Omega}_{0}^{\mathbf{a}} \frac{1}{R^{2}} \bar{\Omega}_{1}^{\mathbf{b}} \\
& +\bar{\Omega}^{\mathbf{a}} \bar{\Omega}^{\mathbf{b}} \frac{\left(r_{\mathrm{e}, \mathbf{a}}+r_{\mathrm{e}, \mathbf{b}}\right)^{2}}{R^{2}\left(R-r_{\mathrm{e}, \mathbf{a}}-r_{\mathrm{e}, \mathbf{b}}\right)}+\epsilon,
\end{aligned}
$$

where the parameters $\bar{\Omega}^{\mathbf{a}}$ and $r_{\mathrm{e}, \mathbf{a}}$ are again required to fulfill Eq. (23) for $l=0, \ldots, \infty$, and an analogous relation defines the pair $\bar{\Omega} \mathbf{b}, r_{\mathrm{e}, \mathbf{b}}$.

With Eqs. (24) and (25), compact expressions for ERI estimates have been derived, which are fast to calculate. They represent a central result of our work, and we will henceforth refer to Eq. (24) as zeroth-order multipole estimate (0-OME) and to Eq. (25) as first-order multipole estimate (1-OME).

Obviously the multipole expansion and thus the 0-OME or 1OME cannot account for the overlap of the charge distributions. Any possible influence of the overlap on the estimate will be assessed numerically in Sec. III B.

\section{B. Characterization of charge distributions}

For practical reasons, a prescription how to find the values $\bar{\Omega}^{\mathbf{v}}$ and $r_{\mathrm{e}, \mathbf{v}}$ with $\mathbf{v}=\mathbf{a}, \mathbf{b}$ for a particular Gaussian product is still to be found, which ensures that the inequality (23) holds for all $l=0, \ldots, \infty$. We start the analysis for a product of two primitive Gaussian basis functions $\phi_{\mu}(\mathbf{r})$ and $\phi_{v}(\mathbf{r})$ with angular momenta $l_{\mu}$ and $l_{v}$, respectively. When choosing the center of expansion identical to the position given by the Gaussian product theorem

$$
\mathbf{v}=\frac{\alpha_{\mu} \mathbf{A}_{\mu}+\alpha_{v} \mathbf{A}_{v}}{\alpha_{\mu}+\alpha_{v}},
$$

the highest nonzero multipole moment is $l_{\max }=l_{\mu}+l_{v}$, where $\mathbf{A}_{\mu}$ and $\mathbf{A}_{v}$ are the position vectors of the primitive Gaussians and $\alpha_{\mu}$ and $\alpha_{v}$ are their exponents. This becomes evident by following the recurrence schemes for the multipole moments, given in the works of Sierka et al. ${ }^{22}$ and of Pérez-Jordá and Yang. ${ }^{23}$ Since only a finite number of $\bar{\Omega}_{l}^{\mathrm{v}}$ exist, it is thus trivial to find appropriate values $\bar{\Omega}^{\mathrm{v}}$ and $r_{\mathrm{e}, \mathbf{v}}$ in the case of charge distributions arising from primitive Gaussian functions.

The situation is more complex for charge distributions arising from contracted Gaussians. The product of two contracted basis functions consisting of $N$ and $M$ primitive basis functions, respectively, contains $N \times M$ primitive distributions. Although all of these charge distributions have a finite number of nonzero multipole moments, their centers of expansion $\mathbf{v}_{i}$ with $i=1, \ldots$, $N \times M$ differ [apart from the trivial case, if $\phi_{\mu}(\mathbf{r})$ and $\phi_{v}(\mathbf{r})$ are located at a common center]. However, it is convenient to evaluate the multipole moments at a common center v. Hence, the multipole moments arising from the charge distributions of primitive Gaussians with centers $\mathbf{v}_{i}$ are shifted using the shift operator

$$
\Omega_{l m}^{\mathbf{v}}=\sum_{j=0}^{l} \sum_{k=-j}^{j} O_{l-j, m-k}\left(\mathbf{v}_{i}-\mathbf{v}\right) \Omega_{j k}^{\mathbf{v}_{i}} .
$$

According to this relation, for any nonzero shift, multipole moments of all orders $l$ appear in the expansion. Nevertheless, due to the known structure of $O_{l m}\left(\mathbf{v}_{i}-\mathbf{v}\right)$ [see Eq. (7)], Eq. (23) can be fulfilled also for all orders $l>l_{\mu}+l_{v}$, by choosing

$$
r_{\mathrm{e}, \mathbf{v}} \geq \max _{i}\left|\mathbf{v}_{i}-\mathbf{v}\right|,
$$

where $i$ denotes the primitive charge distributions. Further details on how to choose the parameters $\bar{\Omega}^{\mathbf{v}}$ and $r_{\mathrm{e}, \mathrm{v}}$ are given in Sec. III. 


\section{DETAILED ANALYSIS}

In this section, we present how the proposed integral estimate is used in an actual Hartree-Fock implementation. We work here with the RIPER ${ }^{24}$ module of the TURBOMOLE code ${ }^{25}$ and its recent extension to perform Hartree-Fock calculations for periodic systems. ${ }^{26}$ We show in Subsection III A how to evaluate the quantities appearing in the screening formulas (23)-(25). A numerical validation of the fact that the ERI estimate is an upper bound to the true ERI is then given in Sec. III B, both exemplarily for two prototypical integrals and in terms of a statistical analysis of all integrals appearing in a test calculation of a deoxyribonucleic acid (DNA) molecule. In Sec. III C, we finally explain how we can further optimize the ERI screening algorithm for the construction of the Fock exchange by preselection of integrals. Throughout this work, charge distributions will be considered to be WS in terms of the relations for extent and separation in Eqs. (5) and (6), using an overlap threshold of $\epsilon_{\mathrm{o}}=10^{-4}$. Furthermore, we will specify almost all quantities in terms of atomic units (a.u.). We will hence indicate units only if they differ from a.u. and suppress them otherwise.

\section{A. Characterization of charge distributions}

The evaluation of the 0-OME or 1-OME in Eqs. (24) and (25) requires a characterization of charge distributions $\mid \mu v)$ and $\mid \kappa \lambda)$ in terms of $\bar{\Omega}^{\mathbf{a}}, r_{\mathrm{e}, \mathbf{a}}$ and $\bar{\Omega}^{\mathbf{b}}, r_{\mathrm{e}, \mathbf{b}}$ in order to use the inequality in Eq. (23). Let us therefore start with the discussion on how to determine these parameters.

If a charge distribution $\phi_{\mu}(\mathbf{r}) \phi_{v}(\mathbf{r})$ shows a non-negligible Schwarz estimate, we define a center a. In our implementation, we determine this position through the Gaussian product theorem, applied to the primitive Gaussians with the smallest exponents. Similarly, the extent parameter $r_{\mathrm{ext}, \mu v}$ for the charge distribution $\left.\mid \mu v\right)$ is determined from Eq. (5) with $\alpha_{\mu \nu}$ being the sum of the smallest primitive exponents. $\bar{\Omega}^{\mathbf{a}}$ and $r_{\mathrm{e}, \mathbf{a}}$ need to be chosen such that Eq. (23) is fulfilled for all $l=0, \ldots, \infty$. In order to guarantee this, $r_{\mathrm{e}, \mathrm{a}}$ must not be smaller than stated in Eq. (28). Finally, the quantities $\Omega_{j}^{\mathbf{a}}$ are calculated for all $l=0, \ldots, l_{\mu}+l_{v}$ through Eq. (21). We start our algorithm by setting $\bar{\Omega}^{\mathbf{a}}=\bar{\Omega}_{0}^{\mathbf{a}}$ in order to fulfill inequality (23) for $l=0$. Now, we check the inequality for all orders $l \leq l_{\mu}+l_{v}$. If the inequality is not fulfilled, either $r_{\mathrm{e}, \mathrm{a}}$ or $\bar{\Omega}^{\mathrm{a}}$ have to be increased. In order to obtain well balanced values for $r_{\mathrm{e}, \mathbf{a}}$ and $\bar{\Omega}^{\mathbf{a}}$, we first gradually increase the value of $r_{\mathrm{e}, \mathbf{a}}$, but only up to $r_{\mathrm{e}, \mathbf{a}}=r_{\mathrm{ext}, \mu v} / 2$. Should this not be sufficient, we increase $\bar{\Omega}^{\mathbf{a}}$ until Eq. (23) is fulfilled for all of the $l$ desired. We exemplify the procedure for pairs $\mid p s)$ and $\mid d p)$ in Table I.

\section{B. Numerical validation of 0-OME and 1-OME}

Let us now validate numerically that the developed 0-OME and 1-OME yield upper bounds to ERIs. Exact values and results of different estimates are shown in Fig. 1 for two ERIs with increasing separation $R$ between left and right charge distributions. The Schwarz estimate yields the same value for all separations. On the other hand, the exact integral value and the multipole estimate exhibit an algebraic decay with increasing separation. In the examples considered,
TABLE I. Characterization of charge distributions through $\bar{\Omega}^{\mathrm{a}}, r_{\mathrm{e}, \mathrm{a}}$ in order to fulfill the inequality in Eq. (23). (ps) is formed from a single $p$-type function with exponent 0.1 and a contracted s-type function with the exponents 10,5 , and 1 , which are separated by a distance of 2 . The biggest shift of a charge distribution of primitive Gaussians is found to be 0.16 . Our procedure ultimately yields $\bar{\Omega}^{\mathrm{a}}=2.32 \times 10^{-1}$, as can be seen in the row for $I=0$, and $r_{\mathrm{e}, \mathrm{a}}=0.23<r_{\mathrm{ext}, p s} / 2=2.6$. $\mid d p$ ) contains a $d$-function with exponent 1 and a $p$-function with exponent 0.3 , which are both located on the same atom. Our algorithm determines $\bar{\Omega}^{\mathrm{a}}=5.71 \times 10^{-1}$ and $r_{\mathrm{e}, \mathrm{a}}=r_{\mathrm{ext}, d p} / 2=1.2$. The respective value of $\bar{\Omega}^{\mathrm{a}}$ can be found in the row for $I=0$.

\begin{tabular}{lcccc}
\hline \hline & \multicolumn{2}{c}{$\mid p s)$} & \multicolumn{2}{c}{$\mid d p)$} \\
\hline Order $l$ & $\bar{\Omega}_{l}^{\mathbf{a}}$ & $\bar{\Omega}^{\mathbf{a}} r_{\mathrm{e}, \mathbf{a}}^{l} / l !$ & $\bar{\Omega}_{l}^{\mathbf{a}}$ & $\bar{\Omega}^{\mathbf{a}} r_{\mathrm{e}, \mathbf{a}}^{l} / l !$ \\
\hline 0 & $2.32 \times 10^{-1}$ & $2.32 \times 10^{-1}$ & 0 & $5.71 \times 10^{-1}$ \\
1 & $5.39 \times 10^{-2}$ & $5.39 \times 10^{-2}$ & $5.79 \times 10^{-1}$ & $6.88 \times 10^{-1}$ \\
2 & $1.26 \times 10^{-3}$ & $6.20 \times 10^{-3}$ & 0 & $4.15 \times 10^{-1}$ \\
3 & $7.28 \times 10^{-5}$ & $4.81 \times 10^{-4}$ & $1.67 \times 10^{-1}$ & $1.67 \times 10^{-1}$ \\
4 & $3.08 \times 10^{-6}$ & $2.79 \times 10^{-5}$ & 0 & $5.04 \times 10^{-2}$ \\
5 & $1.03 \times 10^{-7}$ & $1.29 \times 10^{-6}$ & 0 & $1.21 \times 10^{-2}$ \\
6 & $2.80 \times 10^{-8}$ & $4.99 \times 10^{-8}$ & 0 & $2.44 \times 10^{-3}$ \\
7 & $6.52 \times 10^{-11}$ & $1.65 \times 10^{-9}$ & 0 & $4.21 \times 10^{-4}$ \\
\hline \hline
\end{tabular}

the multipole estimate is clearly outperforming the Schwarz estimate for separations larger than 4 .

Whereas the 0-OME and the 1-OME clearly differ for the ERI shown in Fig. 1(a), both estimates are indistinguishable in Fig. 1(b). This is due to the fact that the studied $(d p \mid p s)$ integral in Fig. 1(a) contains no monopole-monopole interaction (see Table I for the composition of the different moments), which is exploited by the 1-OME but not by the 0-OME. By construction, the 0-OME decays like $1 / R$, which leads to a systematic overestimation of the true integral value in the limit of large separations $R$ in Fig. 1(a). The integral shown in Fig. 1(b) contains a monopole-monopole interaction, which determines the interaction for large separations. Therefore, 0 -OME and 1-OME are virtually identical.

In order to validate the method in a realistic calculation, results are shown for the DNA2 molecule ${ }^{27}$ using the def2-SVP basis set. ${ }^{28}$
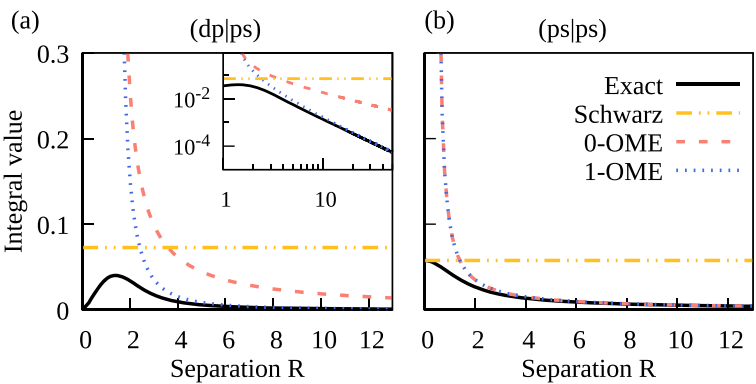

FIG. 1. Exact values and estimates of two different ERIs as a function of the separation $R$ between charge distributions. The estimates consist of the Schwarz estimate together with the 0-OME and 1-OME. The two ERIs (a) (dp|ps) and (b) $(p s \mid p s)$ are formed by the particular distributions introduced in Table I. 


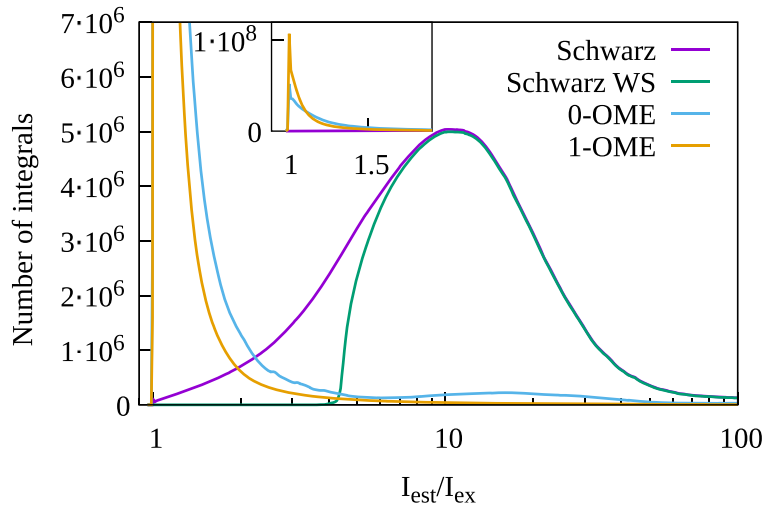

FIG. 2. Histograms for different integral estimates as obtained with the DNA2 molecule. ${ }^{27}$ Shown is the number of integrals for a fixed ratio $l_{\text {est }} / l_{\text {ex }}$ of the integral estimate $\left[l_{\text {est }}=(\mu v \mid \kappa \lambda)_{\text {est }}\right]$ and the exact integral value $\left[l_{\text {ex }}=(\mu v \mid \kappa \lambda)\right]$. The inset displays a magnified view for low ratios.

Figure 2 displays histograms presenting the precision of the Schwarz screening and of the two multipole-based screening methods. For the Schwarz screening, we distinguish between all ERIs and ERIs which are WS. Let us point out that the histograms for 0-OME and 1-OME consider only WS integrals, which passed the Schwarz screening for a threshold of $\epsilon_{\mathrm{s}}=10^{-12}$, since the multipole estimate yields no reliable results for close-by distributions (see also Fig. 1).

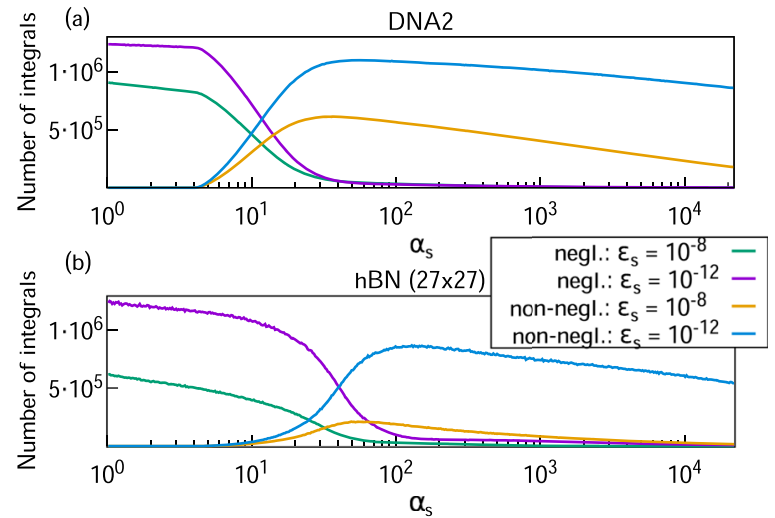

FIG. 3. Number of negligible and non-negligible integrals for different values of $\alpha_{\mathrm{s}}$. Shown are integral batches, which are WS and pass the Schwarz screening. For the analysis, (a) the DNA2 molecule ${ }^{27}$ and (b) $\mathrm{hBN}^{26}$ were used. For the latter, a $27 \times 27 k$-mesh was employed, and the basis sets were def2-SVP ${ }^{28}$ for DNA2 and pob-TZVP ${ }^{29}$ for hBN. For both systems, calculations with two different Schwarz screening thresholds $\left(\epsilon_{\mathrm{s}}=10^{-8}\right.$ and $\left.10^{-12}\right)$ were performed.

The differences between the Schwarz screening for all ERIs and those that are WS show that for close-by distributions the Schwarz estimate is a very accurate upper bound, which usually overestimates the true integral value by no more than a factor of 10 . On the other hand, the splitting of maxima in the histograms for Schwarz
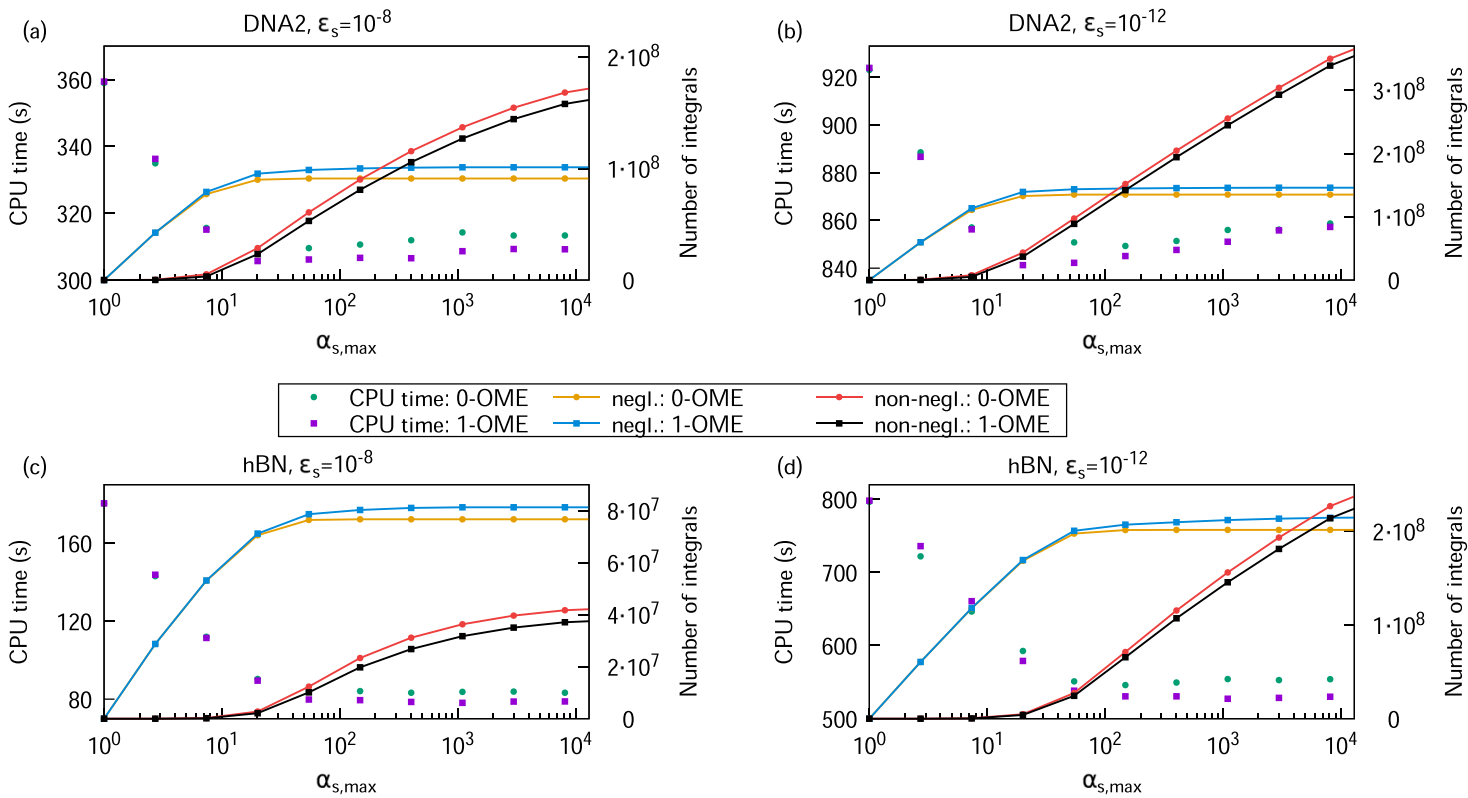

FIG. 4. CPU times and integral statistics are studied for varying values of $\alpha_{s, \max } \geq 1$ for the DNA2 molecule in (a) and (b), and for hBN with $27 \times 27 \mathrm{k}$-points in (c) and (d) with thresholds $\epsilon_{\mathrm{s}}=10^{-8}\left[(\mathrm{a})\right.$ and (c)] and $10^{-12}\left[(\mathrm{~b})\right.$ and (d)]. Shown are CPU times (left axis and points) of the 0-OME (green) and 1-OME (violet) screening schemes. $\alpha_{\mathrm{s}, \text { max }}=1$ is equivalent to pure Schwarz screening, i.e., no additional multipole screening. Solid lines are associated with the right axis and show the number of integral batches, which are identified to be negligible by the 0-OME (yellow) and 1-OME (blue). Similarly, the number of integrals, which are analyzed but are non-negligible, is plotted for 0-OME (red) and 1-OME (black). The basis sets and structures used are the same as given in Fig. 3. 
screening and multipole-based screening illustrates the bad performance of the Schwarz estimate for WS distributions.

We emphasize that no WS ERIs are underestimated by the 0 -OME and 1-OME. This is apparent from Fig. 2, where the histograms vanish for ratios $(\mu v \mid \kappa \lambda)_{\text {est }} /(\mu v \mid \kappa \lambda)<1$. Hence, we can exclude a significant impact of the overlap error on our integral estimates.

\section{ERI preselection for Fock matrix construction}

In our implementation, the multipole estimate is used as an additional screening criterion for all WS ERIs that pass the Schwarz screening. In this sense, the 0-OME and 1-OME screening procedures should be seen as "combined" Schwarz+0-OME or Schwarz+1-OME schemes.

We want to construct the matrix $K_{\mu v}$ of the Fock exchange by using Eq. (1). By defining the quantity

$$
\alpha_{\mathrm{s}}=\frac{\bar{D}(\mu \nu \mid \mu \nu)^{1 / 2}(\kappa \lambda \mid \kappa \lambda)^{1 / 2}}{\epsilon_{\mathrm{s}}}
$$

with $\bar{D}=\max \left\{\left|D_{\mu \kappa}\right|,\left|D_{v \kappa}\right|,\left|D_{\mu \lambda}\right|,\left|D_{\nu \lambda}\right|\right\}$, we can quantify how far the Schwarz-based estimate for the exchange matrix element is away from the target threshold $\epsilon_{\mathrm{s}}$.

In Fig. 2, we saw that the Schwarz estimate overestimates the true value of ERIs in the far field by a factor of roughly 5-100.
Accordingly, additionally screenable integrals are only expected in the range of $1<\alpha_{\mathrm{s}}<100$. In order to test this hypothesis, we classify the ERIs in the following as "negligible" or "non-negligible," if $\alpha=\bar{D}|(\mu \nu \mid \kappa \lambda)| / \epsilon_{\mathrm{s}}<1$ or $\alpha \geq 1$, respectively.

Figure 3 characterizes integrals according to the value of $\alpha_{\mathrm{s}}$ for two systems, namely, DNA2 ${ }^{27}$ and hexagonal boron nitride (hBN). ${ }^{26}$ Since integrals with $\alpha_{\mathrm{s}}<1$ are already screened out by the Schwarz screening, they are not analyzed further. Moreover, only WS integrals are shown because only these are accessible to the multipole screening developed. Independent of the system and the used threshold $\epsilon_{s}$, integrals with low $\alpha_{\mathrm{s}}$ are seen to be negligible with high probability. More precisely, almost all of the negligible integrals are found to coincide with values $\alpha_{\mathrm{s}}<100$. Hence, it seems wise to apply the additional multipole screening only to ERIs below a given $\alpha_{\mathrm{s}, \max }$.

\section{BENCHMARKS AND TIMINGS}

The efficiency gain achievable with the additional multipole screening depends on the investigated system, selected integral neglect threshold $\epsilon_{\mathrm{s}}$, and quantity $\alpha_{\mathrm{s}, \max }$. In the following, $\alpha_{\mathrm{s}, \max }$ will be used as an adjustable parameter, which determines if a particular integral batch is checked by the 0-OME or 1-OME. Only integrals for which $\alpha_{\mathrm{s}}<\alpha_{\mathrm{s}, \max }$ will be examined by the multipole estimate.

Central processing unit (CPU) time and integral statistics for 0 OME and 1-OME are shown in Fig. 4 as a function of the parameter

TABLE II. Performance of the 1-OME screening approach for various systems. Tests are carried out for up to two different thresholds $\epsilon_{\mathrm{S}}=10^{-8}$ and $10^{-12}$. The table lists the absolute number of integral batches, which pass the Schwarz screening ("Ints."), followed by WS integrals ("WS ints.") and WS integrals, which are actually smaller than the target threshold $\epsilon_{\mathrm{S}}$ ("Negl. ints."). The latter two quantities ("WS ints." and "Negl. ints.") are given in percent by putting them into relation to the number of integrals, which pass the Schwarz screening ("Ints."). The next column shows the ratio of actually screened integrals and the total number of negligible WS integrals ("Ident. ints."). The last two columns finally display the proportion of integrals passing the Schwarz screening to integrals actually evaluated after additional 1-OME screening ("Ratio ints.") and the speed-up ("Speed-up"), which is determined as the ratio of CPU times for the Schwarz calculation and the 1-OME scheme. Timings are obtained from the computation of a single exchange matrix. In all of the calculations, ERIs are only tested if $\alpha_{\mathrm{S}}<\alpha_{\mathrm{s}, \max }=100$. Molecular systems are calculated with def-SVP ${ }^{28}$ and def-TZVP ${ }^{31}$ basis sets; for solid-state systems, we use the pob-TZVP basis set. ${ }^{29}$ Molecular structures are taken from Ref. 27, solid state systems from Ref. 26.

\begin{tabular}{|c|c|c|c|c|c|c|c|c|}
\hline & Basis set & $\epsilon_{\mathrm{s}}$ & Ints. $\left(10^{6}\right)$ & WS ints. (\%) & Negl. ints. (\%) & Ident. ints. (\%) & Ratio ints. & Speed-up \\
\hline Benzene & def-SVP & $10^{-8}$ & 0.63 & 10.0 & 0.55 & 52.0 & 1.00 & 1.00 \\
\hline Benzene & def-SVP & $10^{-12}$ & 0.68 & 10.4 & 0.14 & 8.5 & 1.00 & 1.00 \\
\hline Amylose $_{2}$ & def-SVP & $10^{-8}$ & 32.71 & 54.6 & 22.3 & 87.3 & 1.12 & 1.05 \\
\hline Amylose $_{2}$ & def-SVP & $10^{-12}$ & 57.87 & 56.4 & 10.5 & 80.3 & 1.05 & 1.02 \\
\hline DNA2 & def-SVP & $10^{-8}$ & 364.0 & 80.5 & 29.7 & 94.8 & 1.38 & 1.15 \\
\hline DNA2 & def-SVP & $10^{-12}$ & 960.8 & 83.3 & 16.2 & 95.1 & 1.17 & 1.07 \\
\hline DNA2 & def-TZVP & $10^{-8}$ & 3562 & 83.6 & 34.6 & 94.2 & 1.46 & 1.21 \\
\hline DNA2 & def-TZVP & $10^{-12}$ & 9387 & 86.0 & 18.2 & 95.2 & 1.20 & 1.10 \\
\hline $\mathrm{hBN}, N_{k}=13^{2}$ & pob-TZVP & $10^{-8}$ & 60.08 & 94.6 & 49.0 & 97.9 & 1.79 & 1.61 \\
\hline $\mathrm{hBN}, N_{k}=13^{2}$ & pob-TZVP & $10^{-12}$ & 189.6 & 95.8 & 27.2 & 97.9 & 1.33 & 1.27 \\
\hline $\mathrm{hBN}, N_{k}=27^{2}$ & pob-TZVP & $10^{-8}$ & 123.8 & 97.4 & 68.1 & 99.1 & 2.81 & 2.25 \\
\hline $\mathrm{hBN}, N_{k}=27^{2}$ & pob-TZVP & $10^{-12}$ & 563.7 & 98.6 & 39.0 & 99.0 & 1.62 & 1.49 \\
\hline Graphene, $N_{k}=13^{2}$ & pob-TZVP & $10^{-8}$ & 89.59 & 94.3 & 47.0 & 97.9 & 1.73 & 1.56 \\
\hline Graphene, $N_{k}=29^{2}$ & pob-TZVP & $10^{-8}$ & 214.3 & 97.6 & 65.1 & 99.1 & 2.56 & 2.14 \\
\hline Diamond, $N_{k}=9^{3}$ & pob-TZVP & $10^{-8}$ & 1065 & 96.3 & 57.0 & 98.2 & 2.15 & 1.81 \\
\hline Diamond, $N_{k}=13^{3}$ & pob-TZVP & $10^{-8}$ & 1814 & 97.8 & 66.7 & 98.8 & 2.77 & 2.23 \\
\hline Diamond, $N_{k}=17^{3}$ & pob-TZVP & $10^{-8}$ & 2192 & 98.2 & 72.0 & 99.1 & 3.29 & 2.29 \\
\hline
\end{tabular}


$\alpha_{s, \max }$. A central question is the particular speed-up achievable with the multipole screening. The smallest acceleration of 1.1 is obtained for the DNA2 calculation using $\epsilon_{\mathrm{s}}=10^{-12}$. The highest value of 2.3 occurs for hBN and $\epsilon_{\mathrm{s}}=10^{-8}$. To ensure a fair comparison, the timings represent a single construction of the exchange matrix. In all cases, the initial density was taken from a well-converged density functional theory calculation with the Perdew-Burke-Ernzerhof ${ }^{30}$ (PBE) exchange-correlation functional. The large spread of the speed-up can be explained by the number of effectively screened integrals. For the DNA2 molecule with $\epsilon_{\mathrm{s}}=10^{-12}$, only $15 \%$ of all integrals could be identified to be negligible with the multipole screening, avoiding their evaluation. For the hBN calculation with $\epsilon_{\mathrm{s}}=10^{-8}$, this value increases to $68 \%$, which leads to the correspondingly larger acceleration. The comparison between 0-OME and 1-OME is unambiguous. In all cases, the 1-OME scheme shows a faster performance. Nevertheless, the difference is only around $1 \%$.

The integral statistics in Fig. 4 show in all cases that almost all negligible integrals belong to values $\alpha_{\mathrm{s}}<100$. For higher values of $\alpha_{\mathrm{s} \text {,max }}$, only the number of non-negligible integrals increases steadily. Consequently, the CPU time does not decrease any further for $\alpha_{\mathrm{s}, \max }$ $>100$. For DNA2 with $\epsilon_{\mathrm{s}}=10^{-12}$, the CPU time actually increases for $\alpha_{\mathrm{s}, \max }>100$.

Detailed results for several molecules and solid-state systems are summarized in Table II. In these calculations, the 1-OME scheme is employed for WS ERIs with $\alpha_{\mathrm{s}, \max }<100$. The obtained speed-up shows a large spread, ranging from 1.00 to 2.29 , which correlates with the size of the molecule or corresponding solid-state system. The actual speed-up is in all of the cases below the ratio of integrals evaluated with the pure Schwarz screening and the additional multipole screening. Possible reasons could be that there is a certain screening overhead or that the simple proportionality between $\mathrm{CPU}$ time and integral batch does not hold in all of the cases.

Except for the benzene and amylose molecules, the majority of ERIs is WS and the screening scheme identifies more than $94 \%$ of the negligible integrals. Hence, a superior integral estimate for well-separated distributions would not lead to significant improvements over the scheme presented here. The obtained efficiency gain is mainly determined by the number of actually negligible integrals, whose portion increases for larger systems and looser thresholds $\epsilon_{\mathrm{s}}$.

\section{CONCLUSIONS}

The accurate multipole-based ERI estimate presented here is easy to evaluate and can be used in any electronic structure calculations employing local basis sets. It assumes charge distributions to be well separated, i.e., without direct overlap. The overlap error, the difference between the true value of the ERI and the result from the multipole approximation up to infinite order, was studied analytically and numerically. We could exclude a significant underestimation of particular integrals due to the overlap error in numerical tests.

We introduced the new multipole-based ERI estimate in Hartree-Fock calculations as an additional integral screening criterion on top of the Schwarz inequality. The extra number of integrals filtered out as compared to the pure Schwarz screening was studied for a variety of systems ranging from molecules to periodic structures, and the efficiency increase was analyzed. We have shown that the presented screening correctly identifies more than $90 \%$ of the negligible integrals. The analysis also revealed that the multipolebased ERI screening is advantageous for large molecules and for solid-state systems, since in this situation a considerable number of integrals are excluded by the additional multipole screening.

The proposed estimate is universal and could, in principle, be used not only for the Fock term, like in this work, but also for the Hartree term when evaluated with the fast multipole method. Similarly, the formalism could be exploited in the determination of energy gradients instead of total energies. Further applications could be in electronic structure methods treating electron correlation or in local-pair natural-orbital methods, where the multipole method is used for prescreening of weak ${ }^{32}$ or for the evaluation of far-separated ${ }^{8}$ electron pairs. Another obvious area of application would be a precise integral evaluation scheme based on the multipole method. It is straightforward to find expressions like Eqs. (24) and (25), which treat a certain number of multipole moments explicitly and provide knowledge about the ERI contribution of higher multipole moments. This would allow a well controllable integral evaluation via the multipole expansion. Significant speed-ups for ERI evaluation can be expected for highly contracted basis functions as well as for basis functions with high angular momentum $l$. Such an integral evaluation scheme is already used, however without accurate error control. ${ }^{8-10}$

\section{ACKNOWLEDGMENTS}

A.I. and F.P. acknowledge financial support from the Carl Zeiss Foundation as well as the Collaborative Research Center (SFB) 767 of the German Research Foundation (DFG). A.I. thanks the Okinawa Institute of Science and Technology for its hospitality during a one-week-long visit. Part of the numerical modeling was performed using the computational resources of the bwHPC program, namely, the bwUniCluster and the JUSTUS HPC facility.

\section{REFERENCES}

${ }^{1}$ A. Szabo and N. S. Ostlund, Modern Quantum Chemistry: Introduction to Advanced Electronic Structure Theory (Dover, Mineola, 1989).

${ }^{2}$ M. Häser and R. Ahlrichs, J. Comput. Chem. 10, 104 (1989).

${ }^{3}$ C. A. White and M. Head-Gordon, J. Chem. Phys. 101, 6593 (1994).

${ }^{4}$ C. A. White, B. G. Johnson, P. M. Gill, and M. Head-Gordon, Chem. Phys. Lett. 230, 8 (1994).

${ }^{5}$ L. Greengard and V. Rokhlin, J. Comput. Phys. 73, 325 (1987).

${ }^{6}$ T. Helgaker, P. Jørgensen, and J. Olsen, Molecular Electronic Structure Theory (John Wiley \& Sons, Ltd., Chichester, 2000).

${ }^{7}$ J. C. Burant, G. E. Scuseria, and M. J. Frisch, J. Chem. Phys. 105, 8969 (1996).

${ }^{8}$ G. Hetzer, P. Pulay, and H.-J. Werner, Chem. Phys. Lett. 290, 143 (1998).

${ }^{9}$ C. Pisani, R. Dovesi, and C. Roetti, Hartree-Fock Ab Initio Treatment of Crystalline Systems, Lecture Notes in Chemistry Vol. 48 (Springer-Verlag, Berlin, 1988).

${ }^{10}$ E. Schwegler and M. Challacombe, J. Chem. Phys. 111, 6223 (1999).

${ }^{11}$ D. S. Lambrecht and C. Ochsenfeld, J. Chem. Phys. 123, 184101 (2005).

${ }^{12}$ D. S. Lambrecht and C. Ochsenfeld, J. Chem. Phys. 136, 149901 (2012).

${ }^{13}$ S. A. Maurer, D. S. Lambrecht, D. Flaig, and C. Ochsenfeld, J. Chem. Phys. 136, 144107 (2012).

${ }^{14}$ D. S. Hollman, H. F. Schaefer, and E. F. Valeev, J. Chem. Phys. 142, 154106 (2015).

${ }^{15}$ T. H. Thompson and C. Ochsenfeld, J. Chem. Phys. 147, 144101 (2017).

${ }^{16}$ F. Weigend, Phys. Chem. Chem. Phys. 4, 4285 (2002). 
${ }^{17}$ F. Neese, F. Wennmohs, A. Hansen, and U. Becker, Chem. Phys. 356, 98 (2009). ${ }^{18}$ D. G. Liakos, R. Izsák, E. F. Valeev, and F. Neese, Mol. Phys. 111, 2653 (2013).

${ }^{19}$ E. Condon and G. Shortley, The Theory of Atomic Spectra (Cambridge University Press, 1970)

${ }^{20} \mathrm{~T}$. Brink and G. Satchler, Angular Momentum (Oxford University Press, London, 1962).

${ }^{21}$ C. A. White and M. Head-Gordon, J. Chem. Phys. 105, 5061 (1996).

${ }^{22}$ M. Sierka, A. Hogekamp, and R. Ahlrichs, J. Chem. Phys. 118, 9136 (2003).

${ }^{23}$ J. M. Pérez-Jordá and W. Yang, J. Chem. Phys. 107, 1218 (1997).

${ }^{24}$ R. Łazarski, A. M. Burow, and M. Sierka, J. Chem. Theory Comput. 11, 3029 (2015).
${ }^{25}$ F. Furche et al., Wiley Interdiscip. Rev.: Comput. Mol. Sci. 4, 91 (2013).

${ }^{26}$ A. Irmler, A. M. Burow, and F. Pauly, J. Chem. Theory Comput. 14, 4567 (2018).

${ }^{\mathbf{2 7}}$ See http://www.cup.uni-muenchen.de/pc/ochsenfeld/download.html for Molecular structures.

${ }^{28}$ F. Weigend, F. Furche, and R. Ahlrichs, J. Chem. Phys. 119, 12753 (2003)

${ }^{29}$ M. F. Peintinger, D. V. Oliveira, and T. Bredow, J. Comput. Chem. 34, 451 (2012).

${ }^{30}$ J. P. Perdew, K. Burke, and M. Ernzerhof, Phys. Rev. Lett. 77, 3865 (1996).

${ }^{31}$ A. Schäfer, C. Huber, and R. Ahlrichs, J. Chem. Phys. 100, 5829 (1994).

${ }^{32}$ C. Riplinger and F. Neese, J. Chem. Phys. 138, 034106 (2013). 